\title{
New Electromagnetic Force-Displacement Sensor
}

\author{
Amine Benabdellah*, Zakarya Abbassi*, Abdelrhani Nakheli** \\ *Department of Physics, F.S, Laboratory LASMAR, Moulay Ismail University, Meknes, Morocco \\ **Department of Electrical Engineering, EST BP 3103 Toulal, Moulay Ismail University Meknes Morocco
}

\begin{tabular}{l}
\hline \hline Article Info \\
\hline Article history: \\
Received Sep 11, 2015 \\
Revised Nov 22, 2015 \\
Accepted Dec 16, 2015
\end{tabular}

\section{Keyword:}

Accuracy

Coil

Displacement sensor

Electromagnetic force

Measuring instruments

Spring

\section{Corresponding Author:}

Amine Benabdellah,

Department of Physics,

Moulay Ismail University,

Laboratory Lasmar, F.S, Meknes, Morocco.

Email: amine-benabdellah@live.fr

\begin{abstract}
A new electromagnetic force-displacement sensor is presented. Its operating principle is based on the fundamental laws of electromagnetism (FaradayLenz law) and the mechanical properties of a spring. The active elements are two coils made by a wire of $60 \mu \mathrm{m}$ in diameter. Using different wire diameters or different number of wire turns in the coil modify the intensity of the magnetic field and the sensor response. The average accuracy of the sensor is about $\Delta \mathrm{d}=1 \mu \mathrm{m}$, and as a force sensor is about $\Delta \mathrm{F}=1 \mu \mathrm{N}$. This sensor could be successfully used for the manufacture of several measuring instruments.
\end{abstract}

Copyright (c) 2016 Institute of Advanced Engineering and Science. All rights reserved.

\section{INTRODUCTION}

A magnetic sensor is a transducer that converts a magnetic field into a corresponding electrical signal. In general, the principles used in magnetic sensors are numerous; applications are also very different, not only in terms of cost but also in terms of their measurement range and resolution [1-3]. Those inductive sensors which use the inductive effect were first used for historical reasons, but are still interesting because of their accuracy and robustness; thus, inductive sensors are generally used for detection of position, pressure or flaw in mechanical structures.

There are many historical examples of the renaissance of various coil sensors. For example, the Chattock-Rogowski coil was first described in $1887[4,5]$. Today, this sensor has been re-discovered as an excellent current transducer [6] and sensor used in measurement of magnetic properties of soft magnetic materials [7]. An old Austrian patent from 1957 describing the use of a needle sensor (also called the stylus method) for the investigation of local flux density in electrical steel was revived several years ago for magnetic measurements $[8,9]$. Et sans neg

The device we propose is an electromagnetic sensor based on the fundamental laws of electromagnetism and designed for manufacturing of different measuring instruments (Micro-balance, Microdynamometer, Density meter, Viscometer, Dilatometer, Tide gauge, Extensometer, Accelerometer, Seismometer, Gravimeter....)

In this paper, we will expose the operating principle of our implemented sensor and the more adequate configuration after many empirical studies. 


\section{ELECTROMAGNETIC FORCE-DISPLACEMENT SENSOR}

Our goal is to design and produce an electromagnetic sensor with a good accuracy of measurement, stability and specified measuring range and resolution.

The operating principle of the proposed sensor is based on the fundamental laws of electromagnetism (Faraday-Lenz law), which justifies its name "Electromagnetic force sensor".

This sensor consists of two flat circular coils of $1.8 \mathrm{~cm}$ in diameter, placed in parallel. One of the flat coils (Fixed Coil) is fixed on an insulating horizontal support and the other flat coil (Moving Coil) is wound around an insulating cylinder, the lower end passes through the free surface formed by the fixed coil, and the upper end is connected to a spring which is itself attached to a fixed support. At the lower end of the cylinder that acts as a guide, we set a hook for hanging masses. The entire system formed (fixed flat coil, guide cylinder, spring and moving flat coil) is aligned on the same vertical axis (Figure 1).

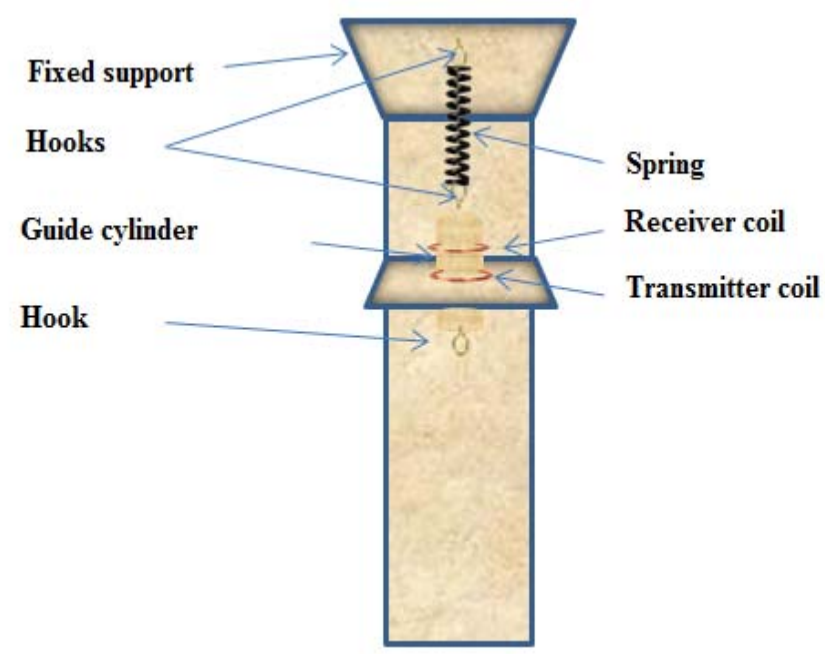

Figure 1. Electromagnetic sensor

The cylinder is movable vertically upwards or downwards virtually without friction, when we exert a force on its lower end, which has the effect of extending or compressing the spring, this action bring closer or away the guide cylinder (moving coil) from the fixed coil.

The fixed coil is supplied by a low frequency generator, and therefore it is traversed by a sinusoidal current which creates a sinusoidal magnetic flux along its axis, the latter creates through the moving coil a variable flow $\Phi$ and induced a measurable sinusoidal electromotive force, the value of the EMF induced depends on the distance $\mathrm{x}$ from the center of the transmitter coil, the flux $\Phi$ is proportional to the magnetic induction $\mathrm{B}$ that changes as a function of $\mathrm{x}$ by the following equation:

$$
B(x)=\frac{\mu_{0} I R^{2}}{2 \sqrt{\left(R^{2}+x^{2}\right)^{3}}}
$$

With I: Maximum amplitude of the current flowing through the coil, $\mathrm{R}$ : coil radius and $\mathrm{x}$ is the distance between the coil center and a point $\mathrm{M}$ on the coil axis.

The magnetic field created by the transmitter coil is at a maximum at the center of the coil $(\mathrm{x}=0)$ :

$$
B_{0}=\frac{\mu_{0} I}{2 R}
$$

The second flat moving coil is the receiver coil; it acts as an inductive sensor that converts a magnetic field flowing there through to a voltage. This receiver coil situated at a distance $\mathrm{x}$ from the fixed flat coil will receive an electromotive force whose expression is given by the Lenz-Faraday law e $=-\mathrm{d} \Phi / \mathrm{dt}$. 
The magnetic flux $\Phi$ through the receiver coil is proportional to the magnetic induction B whose variations in terms of $\mathrm{x}$ are given by the relation (1), and therefore the induced electromotive force will have similar variations in function of $\mathrm{x}$ in a given frequency. The maximum amplitude of this induced current will be greater as the frequency is higher. Thus, we realized an electromagnetic displacement sensor, at each position $\mathrm{x}$ of the receiver coil corresponds a determined induced voltage, this voltage takes its maximum value when the two coils are juxtaposed and decreases as the receiver coil moves away from the transmitter coil. The use of the spring, which acts as a force-displacement converter, allows to use the sensor as a force sensor.

The induced signal is sinusoidal and its amplitude is low, so it has been necessary to introduce circuits for amplification, rectification and filtering to make this voltage usable.

\section{EXPERIMENTAL DEVICE}

The experimental device which allows taking readings of the voltage delivered by the movable coil according to the distance between the two coils is shown in Figure 2.

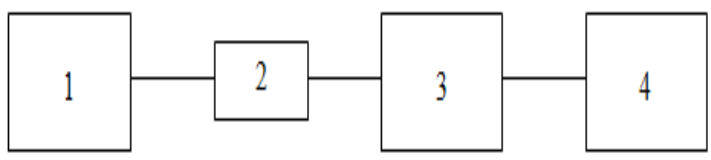

Figure 2. Experimental device: 1-Signal generator; 2-Electromagnetic sensor; 3-Conditioning circuit; 4-Digital voltmeter.

The conditioning circuit includes circuits for amplification, rectification and filtering to make the signal usable, to improve the signal-to-noise ratio and to ensure a better stability (Figure 3 ).

To isolate the sensor from the external perturbation, it was essential to cover our system with a layer of copper to protect it from interfering magnetic fields.

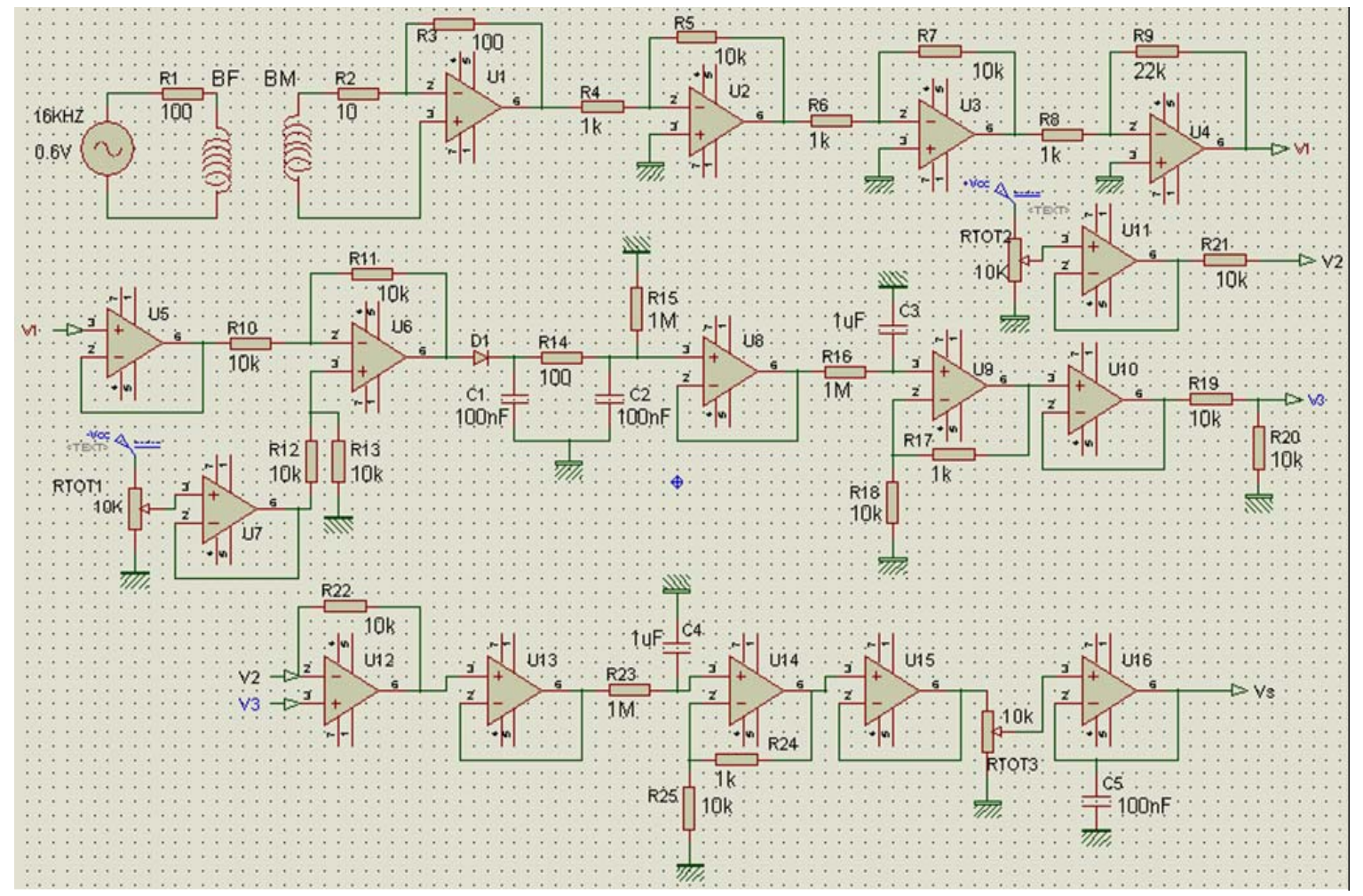

Figure 3. Conditioning circuit 
Amplifiers used are LM324 which are power supplied from a $\pm 15 \mathrm{~V}$ symmetrical stabilized source.

\section{RESULTS AND DISCUSSION}

We have made many experiences by changing many times the number of wire turns and the diameter of coils. We present here the comparison between the responses. First, of two different wire diameters (Figure 4), and secondly, of two different number of wire turns (Figure 5):

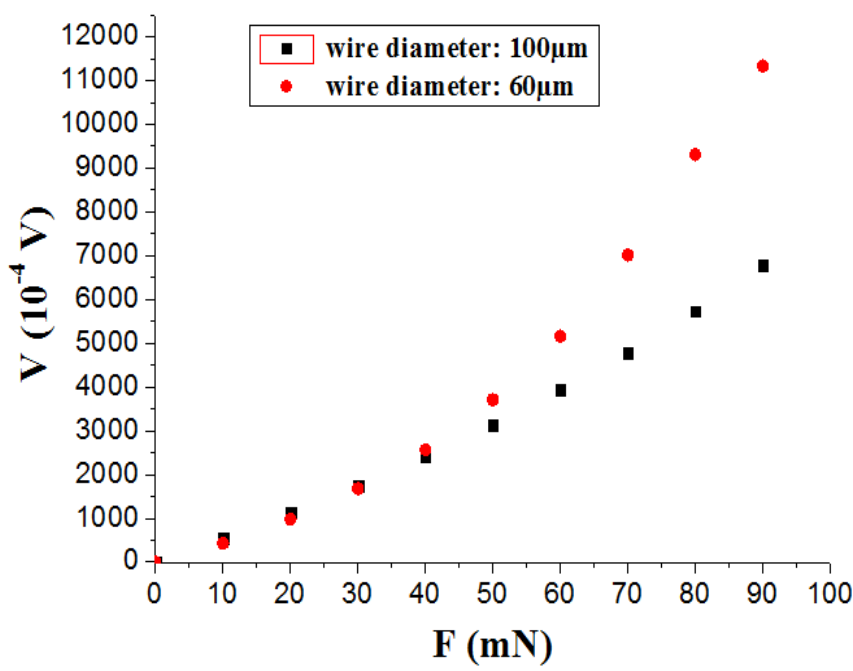

Figure 4. Sensor response for different wire diameters

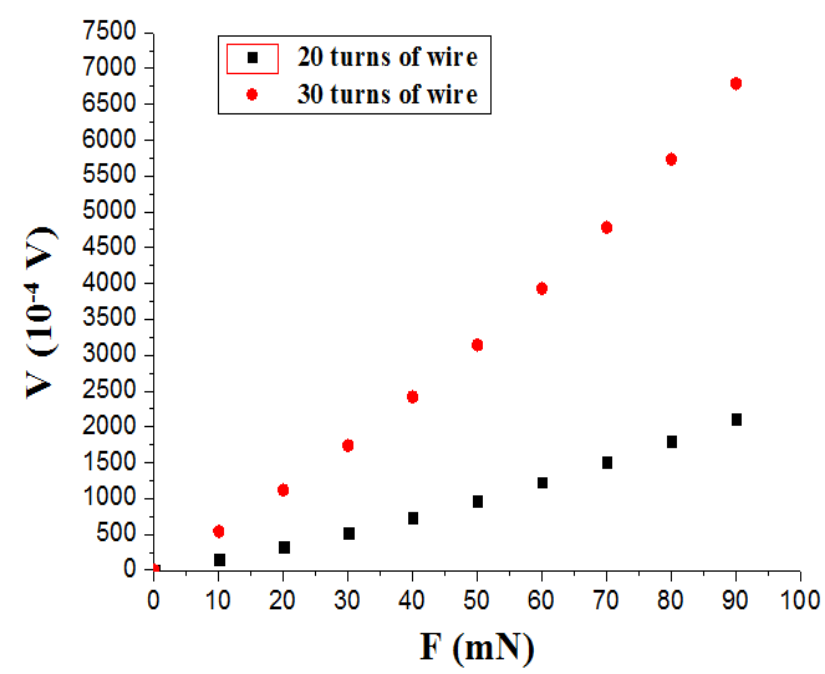

Figure 5. Sensor response for different numbers of wire turns

Since at the receiver side only small signals are expected, it is preferable to increase the number of turns in the pickup coil, even at the cost of a decrease of the cross section of the wire. According to Faraday's law, the number $\mathrm{N}$ of turns in the coil is the relevant factor for the induced voltage [10], but by increasing the number of turns in the coil or the cross section of the wire, it also increases its size which has a negative effect on the signal-to-noise ratio or on the stability of the sensor.

As the accuracy depends obviously on the elements that constitute the experimental device (wire diameter, the number of wire turns, the spring and the signal conditioning circuit), Empirical studies have led us to the discovery of a crucial number of wire turns which is 30 , and a crucial wire diameter which is $60 \mu \mathrm{m}$. 
That gives stability and a good sensitivity comparing to all experiences we made by changing each time the already mentioned parameters. And finally we got the response shown in Figure 6 and Figure 7.

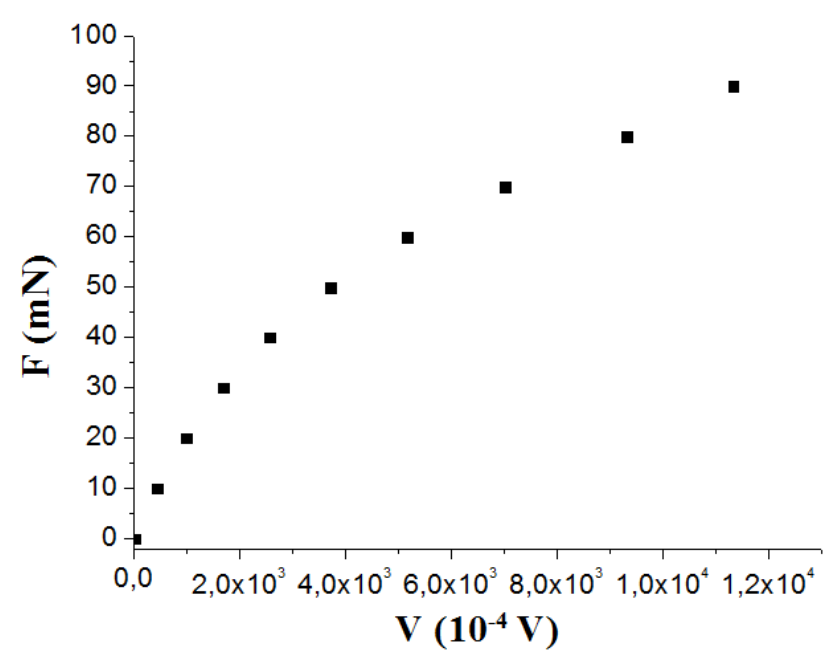

Figure 6. Output voltage variation as a function of force $\mathrm{F}$

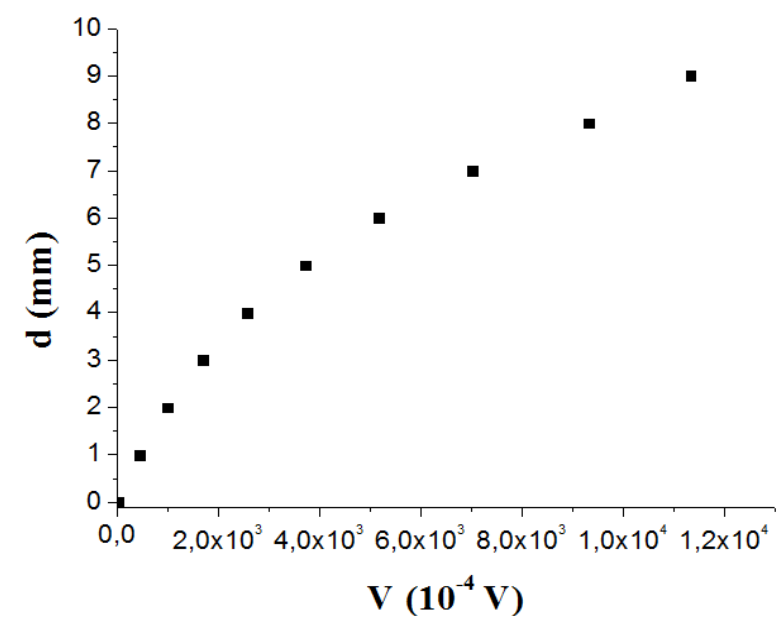

Figure 7. Output voltage variation as a function of displacement $d$

These curves represent the calibration curves, as a force sensor $(F=f(V))$ and as a displacement sensor $(\mathrm{d}=\mathrm{f}(\mathrm{V}))$ after taking readings of the variation of $\mathrm{V}$ as a function of the parameter required.

The sensor response is not linear, it is rather parabolic and follows a polynomial equation, the immediate consequence of this non-linearity is having a variable sensitivity which depends on the distance $\mathrm{x}$ between the two coils according to the equation (1).

The measurement parameters are as follows:

* Sensitivity : The average sensitivity of the sensor depends on the stiffness of the spring used and conditioning circuit. we achieved a sensitivity of $\Delta \mathrm{F}=1 \mu \mathrm{N}$ by using a spring of stiffness $\mathrm{k}=2 \mathrm{~N} / \mathrm{m}$. And as a displacement sensor the sensitivity is $\Delta \mathrm{d}=1 \mu \mathrm{m}$

* Measuring range: By using the sensor as a displacement sensor, the sensor is designed to work in a range of 0 to $1 \mathrm{~cm}$. and by using the sensor as a force sensor it's related to the mechanical properties of the spring (by using for example a spring of stiffness $K=2 \mathrm{~N} / \mathrm{m}$, we have $\Delta \mathrm{F}=1$ $\mu \mathrm{N}$ and the measuring range is from 0 to $1 \mathrm{~g}$ ).

* Accuracy: The average accuracy of the sensor is about $\Delta \mathrm{d}=1 \mu \mathrm{m}$, and as a force sensor is about $\Delta \mathrm{F}=1 \mu \mathrm{N}$.

The accuracy of our sensor is so important and competitive comparing to other available instruments [11-13]. 


\section{CONCLUSION}

We realized a new force-displacement sensor with an important sensitivity and accuracy $(\Delta \mathrm{d}=1 \mu \mathrm{m}$ and $\Delta \mathrm{F}=1 \mu \mathrm{N})$.

This sensor is based on two converters, the first is the moving coil that acts as an inductive sensor that converts a magnetic field passing there through to a voltage, and the second is a spring which allows to calculate the displacement by knowing the value of the force applied and its characteristics (by using for example a spring of stiffness $\mathrm{K}=10 \mathrm{~N} / \mathrm{m}$, the displacement caused by applying a force of $0,01 \mathrm{~N}$ is $1 \mathrm{~mm}$ ). The choice of a good spring (perfectly elastic) is important.

The sensor characteristics depend on the two coils and on the spring. Thus, the effect of the turns number in the coil, the wire diameter and the spring stiffness $\mathrm{k}$ can rather modify the sensitivity and the measuring range of our sensor, and thus, the applications for which it is dedicated.

Applications of this sensor are numerous, and can be used for manufacture of several precision measuring instruments (density meter, dynamometer, viscometer, extensometer, balance, accelerometer, inclinometer, Tide gauge, pressure sensor, mechanical vibration sensor...etc).

Currently, we are developing some of these applications with the data acquisition part using a DAQ card and LabVIEW that is often used in such applications and in the automation of physical systems [14-16].

\section{REFERENCES}

[1] J.E. Lenz. A Review of Magnetic Sensors. Proceedings of the IEEE. 1990; 78(6): 973-989.

[2] J. Lenz, et al. Magnetic Sensors and Their Applications. Sensors Journal IEEE. 2006; 6(3): 631-649.

[3] S. Tumanski. Induction Coil Sensors-a Review. Measurement Science and Technology. 2007; 18(3): R31-R46.

[4] W. Rogowski, W. Steinhaus. Die Messung der magnetischen Spannung (The measurements of magnetic potential). Archiv für Elektrotechnik. 1912; 1(4): 141-150.

[5] A.P. Chattock . On a magnetic Potentiometer. Philosophical Magazine. 1887; 24(146): 94-96.

[6] P. Murgatroyd. Progress with Rogowski coils. EMCWA Conf (Chicago). 1996: 369-374.

[7] G.H. Shirkoohi, A.S. Kontopoulos. Computation of Magnetic Field in Rogowski-Chattock Potentiometer(RCP) compensated magnetic testers. Journal of Magnetism and Magnetic Materials. 1994; 133(1-3): 587-590.

[8] K. Senda, M. Ishida, K. Sato, M. Komatsubara, T. Yamaguchi. Localized Magnetic Properties in Grain-Oriented Electrical Steel Measured by needle Probe Method. Electrical Engineering in Japan. 1999; 126(4): 1-11.

[9] H. Pfützner, G. Krismanic. The needle method for induction tests: sources of error. Magnetics IEEE Transactions. 2004; 40(3): 1610-1616.

[10] R.S. Popovic, J.A. Flanagan, P.A. Besse. The future of magnetic sensors. Sensors and Actuators A. 1996; 56 (1-2): 39-55.

[11] F. Zhu and J.W. Spronck. A Simple Capacitive Displacement Sensor. Sensors and Actuators A. 1991; 26 (1-3): $265-269$

[12] J. Sun, J. Zhang, Z. Lui and G. Zhang. A Vision Measurement Model of Laser Displacement Sensor and its Calibration Method. Optics and Lasers in Engineering. 2013; 51(12): 1344-1352.

[13] DA. Silva et al. A Strain Gauge Tactile Sensor for Finger-Mounted Applications. Instrumentation and Measurement, IEEE Transactions. 2002;51(1): 18-22.

[14] J. Chao, X. Wu-bin and L. Bing. Design of Instrument Control System Based on LebVIEW. TELKOMNIKA Indonesian Journal of Electrical Engineering. 2013; 11(6): 3427-3432.

[15] X. Wang. Design of Temperature Measurement and Data Acquisition System Based on Virtual Instrument LabVIEW. TELKOMNIKA Indonesian Journal of Electrical Engineering. 2014; 12(8): 6027-6035.

[16] Y. Abd Djawad, J. Kiely, P. Wraith and R. Luxton. Lock-in Amplifier as a Sensitive Instrument for Biomedical Measurement; Analysis and Implementation. TELKOMNIKA Indonesian Journal of Electrical Engineering. 2014; 12(10): 7214-7222.

\section{BIOGRAPHIES OF AUTHORS}

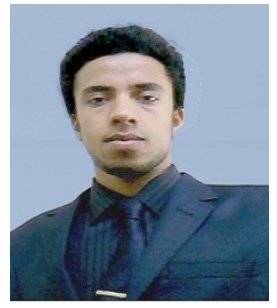

A. Benabdellah received the master degree in 2012 in microelectronics from Ibn-Tofail university, Kenitra, Morocco. Currently he is pursuing his Ph.D. in physics and engineering in Moulay Ismail university of Meknes, Morocco, his work is mainly about the optimization of a new electromagnetic force-displacement sensor, then the design and implementation of several measuring instruments based on this sensor for the study of liquid physical properties. 


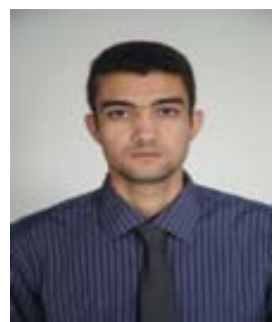

Z. Abbassi received the specialized Master in microelectronics at the Faculty of Sciences, Kenitra, Morocco in 2012. He is currently a Ph.D. student at Department of physics and engineering, Faculty of Sciences Meknes, Morocco. His current field of study is the development of a new generation of electromagnetic force-displacement sensors and its applications.

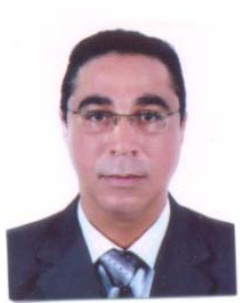

A. Nakheli professor of Higher Education and research scientist in Moulay Ismail University, Meknes, morocco. His research interests is about measurements of liquid physical propreties (density, surface tension, viscosity, intermolecular distances...etc) and development of didactic models based on innovative electromagnetic sensors. 